\title{
Paired related homeobox 1 is associated with the invasive properties of glioblastoma cells
}

\author{
MAI SUGIYAMA ${ }^{1}$, HITOKI HASEGAWA ${ }^{1}$, SATOKO ITO $^{1}$, KAZUYA SUGIYAMA ${ }^{3}$, MASAO MAEDA ${ }^{1}$, \\ KOSUKE AOKI $^{2}$, TOSHIHIKO WAKABAYASHI ${ }^{2}$, MICHINARI HAMAGUCHI ${ }^{1}$, \\ ATSUSHI NATSUME ${ }^{2}$ and TAKESHI SENGA ${ }^{1}$ \\ ${ }^{1}$ Division of Cancer Biology, and Departments of ${ }^{2}$ Neurosurgery and ${ }^{3}$ Obstetrics and Gynecology, \\ Nagoya University Graduate School of Medicine, Showa-ku, Nagoya 466-8550, Japan
}

Received October 13, 2014; Accepted November 13, 2014

DOI: $10.3892 /$ or.2014.3681

\begin{abstract}
Glioblastoma is a highly proliferative and invasive tumor. Despite extensive efforts to develop treatments for glioblastoma, the currently available therapies have only limited effects. To develop novel strategies for glioblastoma treatment, it is crucial to elucidate the molecular mechanisms that promote the invasive properties of glioblastoma. In the present study, we showed that the paired related homeobox 1 (PRRX1) is associated with glioblastoma cell invasion. The depletion of PRRX1 suppressed the invasion and neurosphere formation of glioblastoma cells. Conversely, the exogenous expression of PRRX1 promoted invasion. The Notch signaling pathway, which is an evolutionarily conserved pathway that is essential for developmental processes, plays an important role in the tumorigenesis of glioblastoma. The expression of PRRX1 induced the activation of Notch signaling, and the inhibition of Notch signaling suppressed PRRX1-mediated cell invasion. Our results indicate that activation of Notch signaling by PRRX1 is associated with the promotion of glioblastoma cell invasion.
\end{abstract}

\section{Introduction}

Glioblastoma is one of the most fatal human cancers and is characterized by a high proliferative rate and aggressive invasiveness. Despite advances in surgery, chemotherapy and radiotherapy, no significant increase in survival has been achieved for patients with glioblastoma over the last 20 years. The survival of most glioblastoma patients is less than 2 years $(1,2)$. To develop novel treatments to improve the prognosis of glioblastoma patients, it will be important to further elucidate the molecular mechanisms that support invasion and proliferation of these cancer cells.

Correspondence to: Professor Takeshi Senga, Division of Cancer Biology, Nagoya University Graduate School of Medicine, 65 Tsurumai-cho, Showa-ku, Nagoya 466-8550, Japan

E-mail: tsenga@med.nagoya-u.ac.jp

Key words: glioblastoma, homeobox, PRRX1, invasion, Notch
Homeobox transcriptional factors are a family of proteins with a homeobox domain that binds to conserved DNA sequences. Homeobox proteins are known to play pivotal roles in the developmental processes of multicellular organisms, and accumulating evidence has revealed that certain homeobox genes regulate the progression of numerous types of cancers (3). For example, SIX homeobox 1 (SIX1) is overexpressed in human breast cancers, and transgenic mice that overexpressed SIX1 in mammary epithelial cells exhibited increased tumor development (4,5). A recent study reported that aristaless-like homeobox 1 (ALX1) promoted the invasion of ovarian cancer cells by inducing the epithelial-to-mesenchymal transition (EMT), which is a morphological conversion of epithelial to mesenchymal cells (6). In glioblastoma, HOXA9 expression increased cell proliferation and inhibited apoptosis, and its expression was associated with poor prognosis (7). These studies have shown the important functions of homeobox proteins in tumor progression and support the notion that the inhibition of tumor-associated homeobox proteins can be a novel therapeutic strategy.

Paired related homeobox 1 (PRRX1) is a member of the paired-type family of homeobox transcription factors, which have important functions in the regulation of developmental morphogenetic processes $(8,9)$. PRRX1 is highly expressed in cardiac and skeletal muscle of mouse embryos as well as in adults, and mice lacking PRRX1 die perinatally due to craniofacial and limb malformations (10). PRRX1 is also associated with tumor progression. A high level of PRRX1 expression is correlated with metastasis and poor prognosis of colon cancer cells (11). In contrast, Ocaña et al reported that PRRX1 expression suppressed metastasis and that strong PRRX1 expression could be a marker for good prognosis of breast and lung cancer (12). Although their conclusions are contradictory, both groups reported that PRRX1 expression promotes the EMT in cancer cells. A recent report showed that PRRX1 associates with sex determining region-Y box 2 (SOX2) and is required to maintain the stemness of neuronal stem cells (13). SOX2 has been reported to be overexpressed in numerous types of tumors and to promote invasion of glioblastoma cells (14-17). In the present study, we examined the role of PRRX1 in glioblastoma cells and showed that PRRX1 plays an important role in the invasion of glioblastoma cells. 


\section{Materials and methods}

Cells and antibodies. The human glioblastoma cell lines T98, U251MG, U251sp, U87, SKMG1, U251nu/nu and AO2 and the human embryonic kidney 293T (HEK293T) cell line were maintained in Dulbecco's modified Eagle's medium (DMEM) supplemented with 10\% FBS (Equitech-Bio, Kerrville, TX, USA) at $37^{\circ} \mathrm{C}$ in a humidified atmosphere of $5 \% \mathrm{CO}_{2}$. U251sp and U251nu/nu cells are derived from U251MG cells (18). Anti-E-cadherin, anti-N-cadherin and anti-vimentin antibodies were obtained from BD Biosciences (San Jose, CA, USA) and the anti- $\beta$-actin antibody was from Sigma-Aldrich (St. Louis, MO, USA). The anti-HES1 antibody was purchased from Cell Signaling Technology, Inc. (Danvers, MA, USA).

siRNA transfection. The sequences of the siRNAs specific for PRRX1 consisted of 5'-GCACUAAAGUCUACAUCUA-3' (siPRRX-1) and 5'-CCACUGUUCUUAUCUCUAU-3' (siPRRX-2). The sequence of the control siRNA that targeted luciferase was 5'-CUUACGCUGAGUACUUCGATT-3'. siRNAs were obtained from Sigma-Aldrich. Cells were transfected with $20 \mathrm{nM}$ of siRNA using Lipofectamine RNAiMAX (Invitrogen, Carlsbad, CA, USA) according to the manufacturer's instructions.

Quantitative RT-PCR. RNA was extracted from glioma samples and cells using the RNeasy Mini kit (Qiagen, Venlo, The Netherlands), and cDNA was generated using PrimeScript reverse transcriptase (Takara, Tokyo, Japan). Glioma samples were obtained from patients at Nagoya University Hospital with informed consent. Normal human brain RNAs were obtained from Takara and BioChain (Newark, CA, USA). Catalog nos. of the normal samples are 636530 (Takara) and R1234035-50 (BioChain). PCR was performed using the SYBR Premix Ex $T a q^{\mathrm{TM}}$ II, and the Thermal Cycler Dice ${ }^{\mathrm{TM}}$ Real-time System TP800 (both from Takara) was used for analysis. The relative mRNA expression levels were normalized to GAPDH. The sequences of primers used to amplify each gene were: 5'-AGGTGGAGGAGTGGGTGTCGCTG TT-3' and 5'-CCGGGAAACTGTGGCGTGATGG-3' (GAPDH); 5'-AGTTCCGCAGGAATGAGAGA-3' and 5'-AT GGCGCTTTTCAGTGTCTT-3' (PRRX1A); 5'-CATCGTACC TCGTCCTGCTC-3' and 5'-GCCCCTCGTGTAAACAAC AT-3' (PRRX1B); and 5'-AGGCGGACATTCTGGAAATG-3' and 5'-TCGTTCATGCACTCGCTGA-3' (HES1).

Generation of stable cell lines. To generate T98 and U251MG cells that constitutively expressed GFP, GFP-PRRX1A and GFP-PRRX1B, 293T cells were transfected with a pQCXIP vector (Clontech Laboratories, Inc., Mountain View, CA, USA) encoding each gene as well as the pVPack-GP and pVPackAmpho vectors (Stratagene, Tokyo, Japan). The culture supernatant was collected $48 \mathrm{~h}$ later and applied to T98 or U251MG cells with $2 \mu \mathrm{g} / \mathrm{ml}$ of Polybrene (Sigma-Aldrich). The cells were cultured for $24 \mathrm{~h}$, and then $1 \mu \mathrm{g} / \mathrm{ml}$ of puromycin (Sigma-Aldrich) was added to select for infected cells. T98 and U251MG cells that constitutively expressed dominantnegative RBPJ (DN-RBPJ) with PRRX1 were produced by infecting PRRX1-expressing cells with recombinant retrovirus that encoded DN-RBPJ. The infected cells were selected with neomycin for 5 days. To generate U87 and U251sp cells that constitutively expressed the shRNAs, oligonucleotides encoding shRNAs specific for human PRRX1 and luciferase were cloned into the pSIREN-RetroQ vector (Clontech Laboratories, Inc.). The sequences of the shRNAs were: 5'-GCTTGAAGCTACAGATTAT-3' (shPRRX1), 5'-CCACTG TTCTTATCTCTAT-3' (shPRRX2) and 5'-CTTACGCTGAG TACTTCGA-3' (control). Recombinant retrovirus was produced, and infected U87 and U251sp cells were selected with $1 \mu \mathrm{g} / \mathrm{ml}$ puromycin for 3 days.

Invasion assay. To measure cell invasion using Boyden chambers, a filter was pre-coated with Matrigel, and $4.5 \times 10^{4}$ cells (T98, U251sp, U87) or $1.2 \times 10^{4}$ cells (U251MG) were seeded into the upper surface of the chamber. Twenty hours after seeding, the cells were fixed with $70 \%$ methanol and stained with $0.5 \%$ crystal violet. Cells that invaded the lower surface of the filters were counted in five randomly selected fields. Three independent experiments were performed. To evaluate cell invasion in the presence of the Notch inhibitor, DAPT, cells were treated with DMSO or DAPT $(10 \mu \mathrm{M})$ for $12 \mathrm{~h}$ and then subjected to an invasion assay in the presence of DMSO or DAPT.

Neurosphere assay. Cells were cultured in a 6-well, ultra-low attachment plate (Iwaki, Tokyo, Japan) with DMEM/F-12 medium supplemented with $10 \mathrm{ng} / \mathrm{ml} \mathrm{bFGF}$ and $20 \mathrm{ng} / \mathrm{ml}$ EGF (both from PeproTech, Rocky Hill, NJ, USA). Two weeks later, the number of spheres that were $>50 \mu \mathrm{m}$ in diameter was counted in 10 different, randomly selected fields.

Reporter assay. Cells were transfected with a reporter construct with pRTK-Luc to normalize to the transfection efficiency. Reporter constructs for Notch (19) and Hedgehog signaling (20) were obtained from RIKEN BioResource Center, and a construct for Wnt signaling (21) was obtained from Addgene (Addgene plasmid 12456). Twenty-four hours after transfection, the activities of firefly and Renilla luciferase were measured using the dual-luciferase reporter assay system (Promega, Madison, WI, USA). Luciferase activity was measured in triplicate, and three independent experiments were performed.

Animal experiments. Animal experiments were conducted in accordance with the Faculty of Medicine of Nagoya University. U87 cells expressing control or PRRX1 shRNAs $\left(3.5 \times 10^{5}\right.$ cells $\left./ 5 \mu \mathrm{l}\right)$ were stereotactically injected into the brains of Balb-c nu/nu nude mice (female, 5 weeks old) under anesthesia using a Hamilton syringe (Hamilton, Reno, $\mathrm{NV}$, USA). The coordination was $1.4 \mathrm{~mm}$ posterior from the bregma, $3.0 \mathrm{~mm}$ to the right and at a $4.0-\mathrm{mm}$ depth from the brain surface.

\section{Results}

PRRX1 is expressed in the glioma samples. We first evaluated the mRNA levels of PRRX1 in multiple low-grade and high-grade glioma samples. Two alternative forms of PRRX1, PRRX1A and PRRX1B, exist; both isoforms have a homeobox domain in their central regions, yet they differ at 
A

PRRX1A
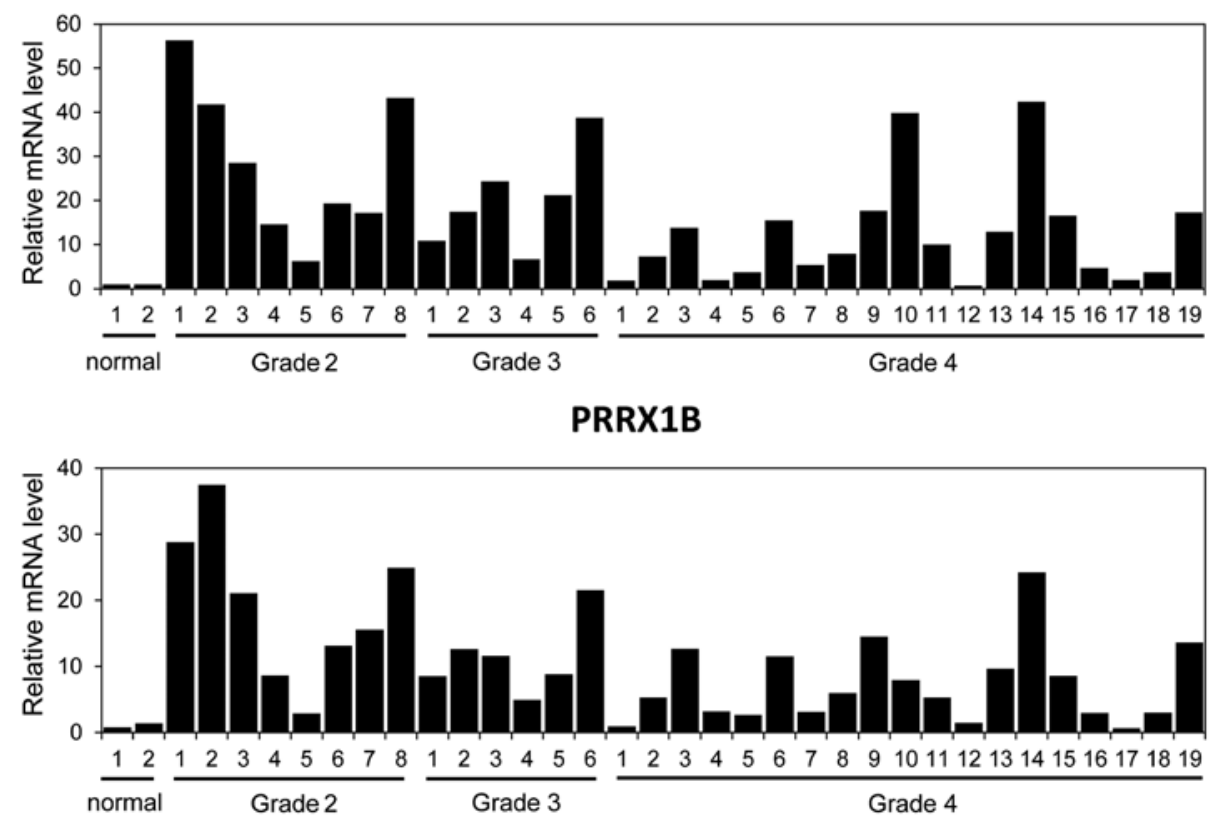

B
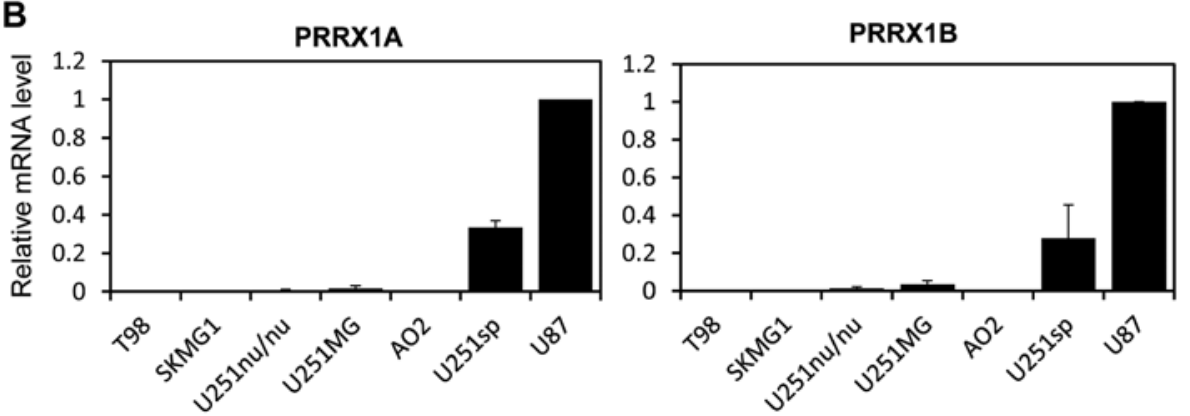

Figure 1. PRRX1 is expressed in glioma samples. (A) Expression of PRRX1A or PRRX1B mRNAs in glioma and normal brain samples was evaluated by quantitative RT-PCR and normalized to the level of GAPDH mRNA. Normal brain sample 1 and 2 were obtained from Takara and BioChain, respectively. (B) Expression of the PRRX1A or PRRX1B mRNAs in glioblastoma cells was evaluated by quantitative RT-PCR and normalized to the level of GAPDH mRNA. PRRX1, paired related homeobox 1 .

their C-termini. PRRX1B has a so-called otp, aristaless and $\operatorname{rax}(\mathrm{OAR})$ domain at its $\mathrm{C}$-terminus, whereas the $\mathrm{C}$-terminal end of PRRX1A lacks this domain. Real-time PCR analysis revealed that the mRNA levels of both isoforms were increased in most of the low-grade and high-grade glioma samples when compared to the levels in the normal brain tissue (Fig. 1A). We next examined expression of PRRX1 in several glioblastoma cell lines. PRRX1A and PRRX1B were highly expressed in the U251sp and U87 cells compared with that in the other glioblastoma cell lines (Fig. 1B).

Silencing of PRRX1 inhibits invasion and neurosphere formation. To examine the role of PRRX1 in glioblastoma cells, we used two different shRNAs that targeted both isoforms of PRRX1. U251sp and U87 cells that constitutively expressed the control (shCtrl) or PRRX1 shRNA (shPRRX-1 and shPRRX-2) were established by retrovirus infection. RT-PCR analysis confirmed a significant reduction in the PRRX1 mRNA in the shPRRX-1 and shPRRX-2 cells (Fig. 2A). Although a previous study reported that PRRX1 is associated with EMT, we did not observe any changes in the expression of EMT markers, such as E-cadherin and vimentin, following PRRX1 knockdown (Fig. 2B). We examined the invasion of PRRX1knockdown cells using Matrigel-coated Boyden chambers. The invasion of U251sp and U87 cells was significantly reduced by PRRX1 knockdown (Fig. 2C). We performed in vitro neurosphere forming assays to assess the tumorigenicity of the PRRX1-depleted cells. Cells were cultured in suspension with EGF and FGF in the absence of serum for 2 weeks, and then the number of neurospheres was determined. As shown in Fig. 2D, neurosphere formation was suppressed by PRRX1 depletion. We then examined the effect of PRRX1 suppression on the proliferation of glioblastoma cells in vivo. Both shCtrl and shPRRX-1 U87 cells were implanted in the brains of mice by intracranial injection, and the survival of the mice was observed. Mice implanted with the PRRX1-knockdown cells exhibited a significantly longer survival when compared with the survival in the shCtrl cell-injected mice (Fig. 2E). These results indicate that PRRX1 is associated with invasion and proliferative properties of glioblastoma cells. 

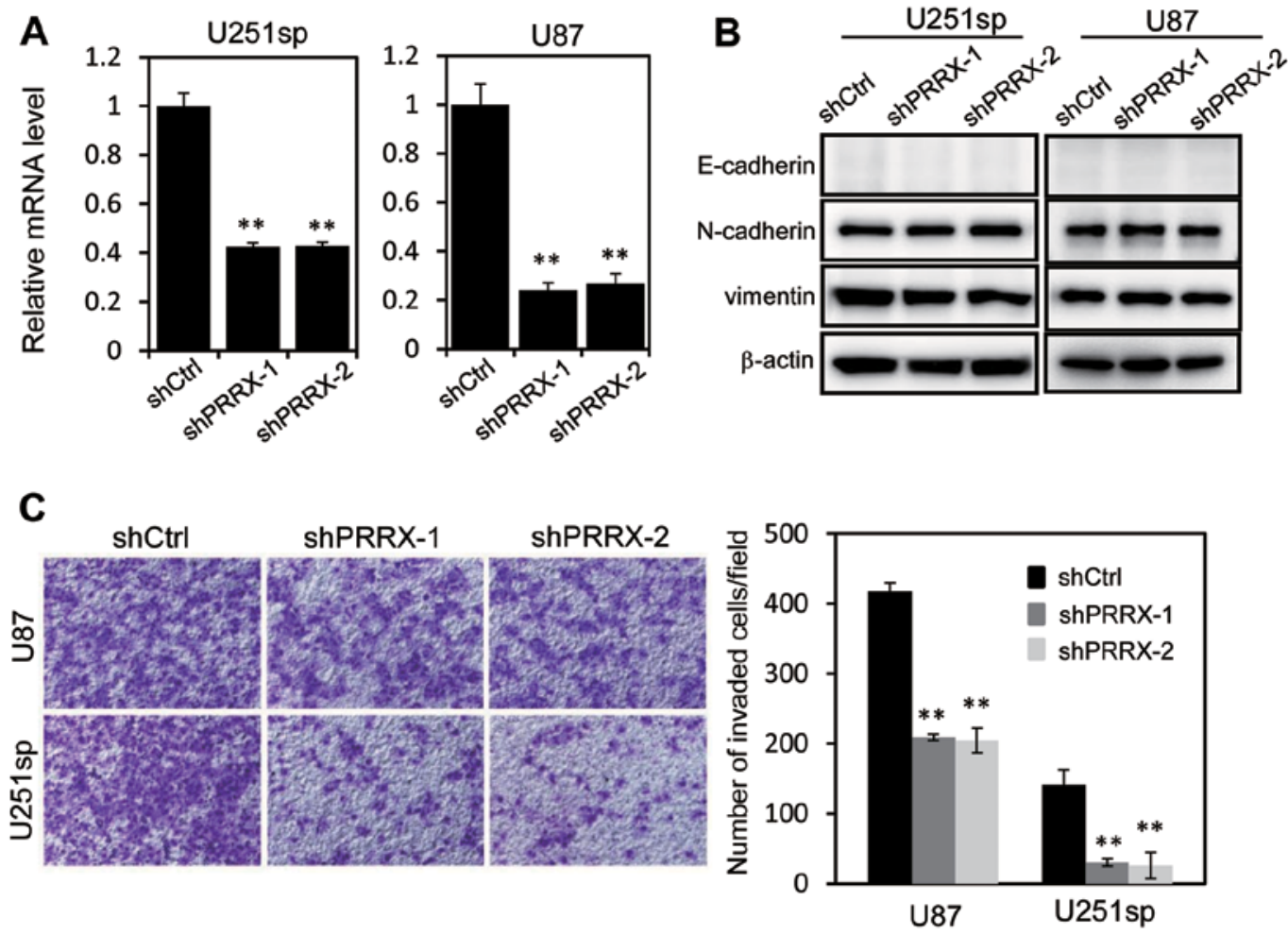

D

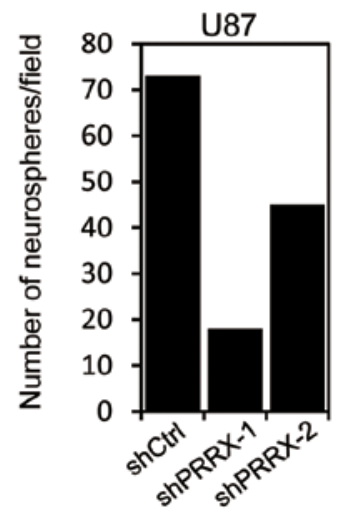

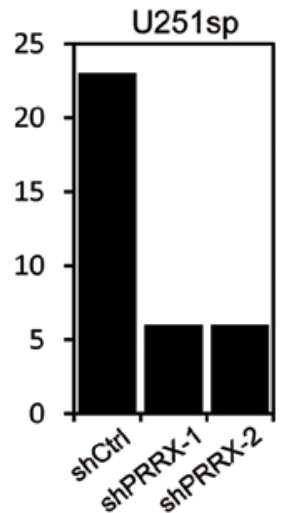

$\mathbf{E}$

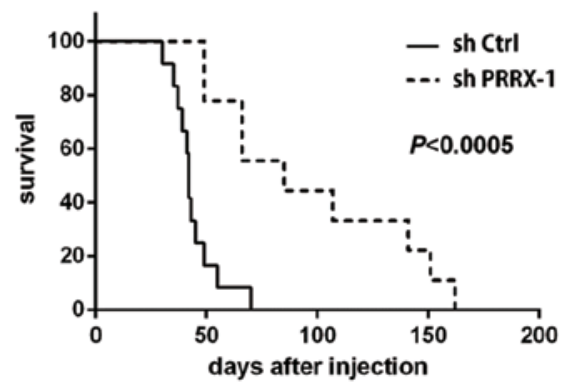

Figure 2. PRRX1 knockdown suppresses the invasion and neurosphere formation of glioblastoma cells. (A) U87 and U251sp cells that constitutively expressed shRNAs were established by retrovirus infection. RNA was extracted from each cell line, and the level of PRRX1 mRNA was determined by quantitative RT-PCR. Three independent experiments were performed, and the data are shown as the means \pm SD $\left({ }^{* *} \mathrm{P}<0.01\right)$. (B) Expression of the indicated proteins was examined by immunoblotting. (C) Cells were subjected to invasion assay. Representative images of invaded cells are shown. The graph indicates the average number of invaded cells/field. Three independent experiments were performed, and the data are shown as the means \pm SD $\left({ }^{* *} \mathrm{P}<0.01\right)$. (D) Cells were cultured in suspension with EGF and FGF in the absence of serum. Two weeks later, the size of each neurosphere was evaluated, and the graph shows the number of neurospheres whose diameters were $>50 \mu \mathrm{m}$. (E) Survival curves of mice injected with shCtrl and shPRRX-1 U87 cells in the brain. PRRX1, paired related homeobox 1 .

Exogenous expression of PRRX1 promotes invasiveness in glioblastoma cells. To further confirm the effects of PRRX1 expression on the invasion of glioblastoma cells, we expressed both isoforms of PRRX1 in T98 and U251MG cells, which showed lower expression of PRRX1 compared with U87 and U251sp cells. T98 and U251MG cells were infected with recombinant virus that encoded GFP-tagged PRRX1A or PRRX1B and selected with puromycin for 2 days. Most of the selected cells clearly expressed GFP-PRRX1, and both isoforms localized to the nucleus (Fig. 3A). We then examined the invasion of PRRX1-expressing cells using Matrigel-coated Boyden chambers. The expression of either isoform of PRRX1 promoted the invasion of T98 and U251MG cells (Fig. 3B). Neurosphere formation assays were also performed to assess tumorigenicity, yet neither GFP- nor GFP-PRRX1-expressing cells formed neurospheres (data not shown).

PRRX1 induces Notch activation. We next investigated the molecular mechanisms by which PRRX1 promotes the invasion of glioblastoma cells. A number of pathways, including the Notch, Wnt and Hedgehog pathways, are activated in glioblastoma cells and are known to be associated with the progression of glioblastoma (22-24). We tested whether these pathways were activated by PRRX1 expression using 
A
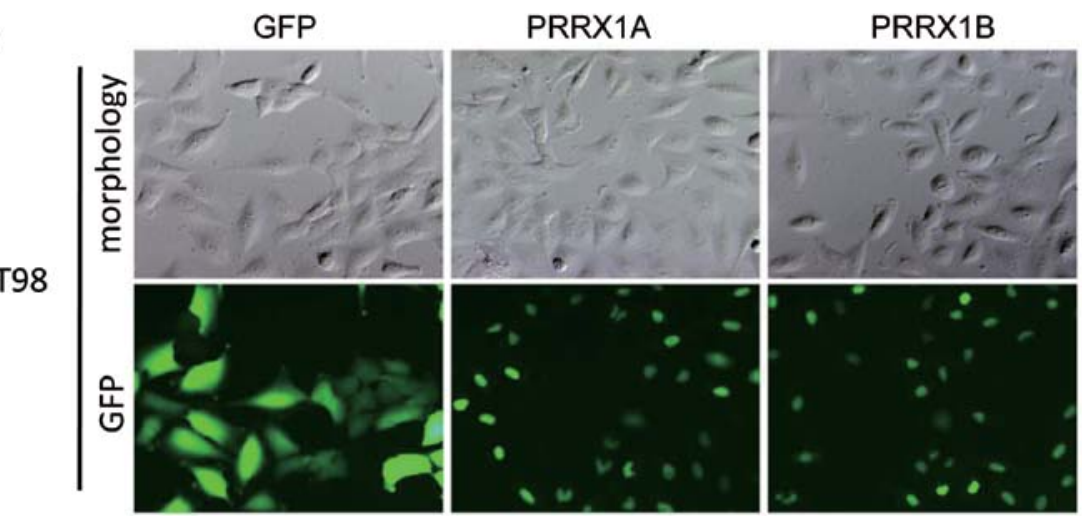

B
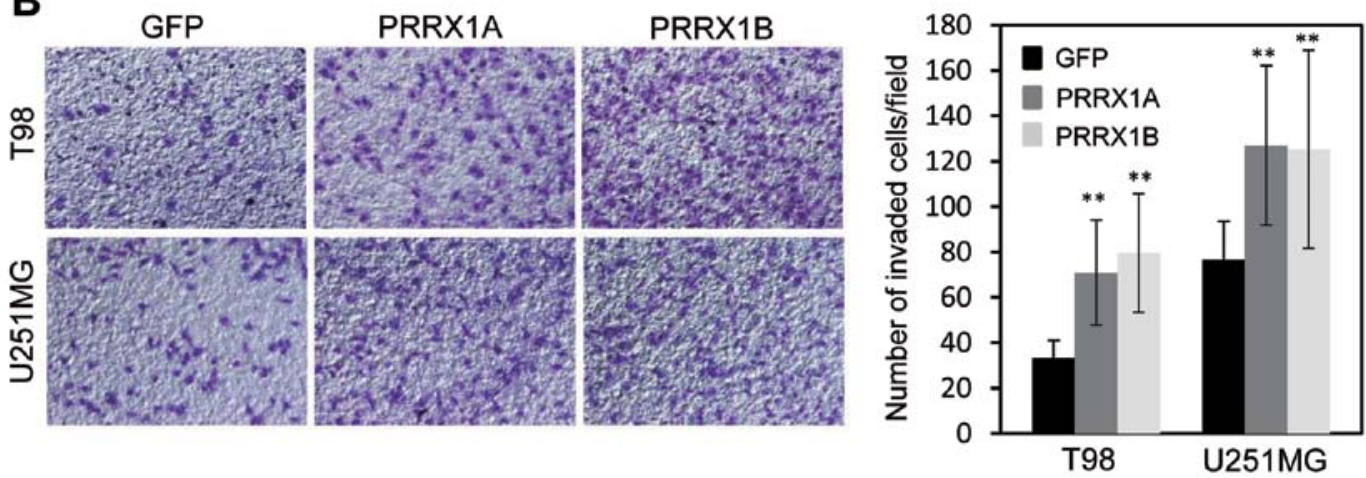

Figure 3. Exogenous expression of PRRX1 promotes invasion. (A) T98 cells were infected with a recombinant retrovirus that encoded GFP, GFP-tagged PRRX1A or PRRX1B, and 3 days later, the cells were selected with puromycin for an additional 2 days. Representative images of infected cells are shown. (B) Cells were subjected to invasion assays, and representative images of invaded cells are shown. The graph indicates the average number of invaded cells/ field. Three independent experiments were performed and the data are shown as the means $\pm \mathrm{SD}\left({ }^{* *} \mathrm{P}<0.01\right)$. PRRX1, paired related homeobox 1 .

a reporter assay. GFP- or GFP-PRRX1-expressing T98 cells were transfected with a reporter plasmid encoding luciferase that could be activated by either pathway, and the luciferase activity was measured $24 \mathrm{~h}$ later. In this analysis, we found specific activation of the Notch pathway in PRRX1-expressing cells (Fig. 4A). To further confirm Notch activation by PRRX1 expression, we examined the level of HES1, which is a target gene of Notch signaling. Consistent with the activation of Notch signaling, increased levels of HES1 mRNA and protein by PRRX1 expression were observed (Fig. 4B and C). Conversely, depletion of PRRX1 expression in U251MG cells by siRNA transfection suppressed HES1 mRNA levels (Fig. 4D). We also tested whether suppression of PRRX1 affected Notch signaling in U87 and U251sp cells, but we did not observe any changes in Notch activation after PRRX1 knockdown (data not shown). It appears that PRRX1-mediated activation of Notch signaling is dependent upon the cellular context. We next examined whether PRRX1 expression was related with HES1 expression in the glioma samples. The expression levels of the PRRX1A and HES1 mRNAs were evaluated in glioma samples using RT-PCR analysis. As shown in Fig. 4E, we observed a correlation between PRRX1A and HES1 expression.

Promotion of invasion by PRRX1 is dependent on Notch pathway activation. We tested whether Notch activation was responsible for the PRRX1-mediated promotion of cell invasion. To inhibit Notch activation, we used a chemical inhibitor, DAPT. Cells were incubated with DAPT for $12 \mathrm{~h}$ and subjected to the invasion assay in the presence of DAPT. Addition of DAPT clearly suppressed the invasion of PRRX1-expressing T98 cells (Fig. 5A). We also used a dominant negative form of the recombination signal binding protein for immunoglobulin kappa J region (RBPJ), which is a transcriptional regulator that is critical for the Notch signaling pathway (25). We established T98 and U251MG cells that constitutively expressed PRRX1 and a dominant-negative form of RBPJ (DN-RBPJ) and examined cell invasion. As shown in Fig. 5B, expression of the DN-RBPJ suppressed the invasion of PRRX1-expressing cells.

\section{Discussion}

In the present study, we studied the role of PRRX1 in glioblastoma cells. Depletion of PRRX1 in U87 and U251sp cells reduced the invasive potential of cells. In addition, tumor implantation experiments showed longer survival of mice injected with PRRX1-knockdown cells when compared with the survival of mice injected with the control cells. Conversely, exogenous expression of PRRX1 in the T98 and U251MG cells clearly promoted cellular invasion. These results clearly indicate that PRRX1 has the potential to promote invasion of glioblastoma. A previous study reported that PRRX1 is an EMT inducer that promotes cancer cell invasion (12). During EMT, non-motile epithelial cells dissolve cell-cell junctions and become motile, invasive mesenchymal cells; therefore, EMT is an important step for cancer cells to acquire invasive potential (26). Although we examined whether PRRX1-mediated 

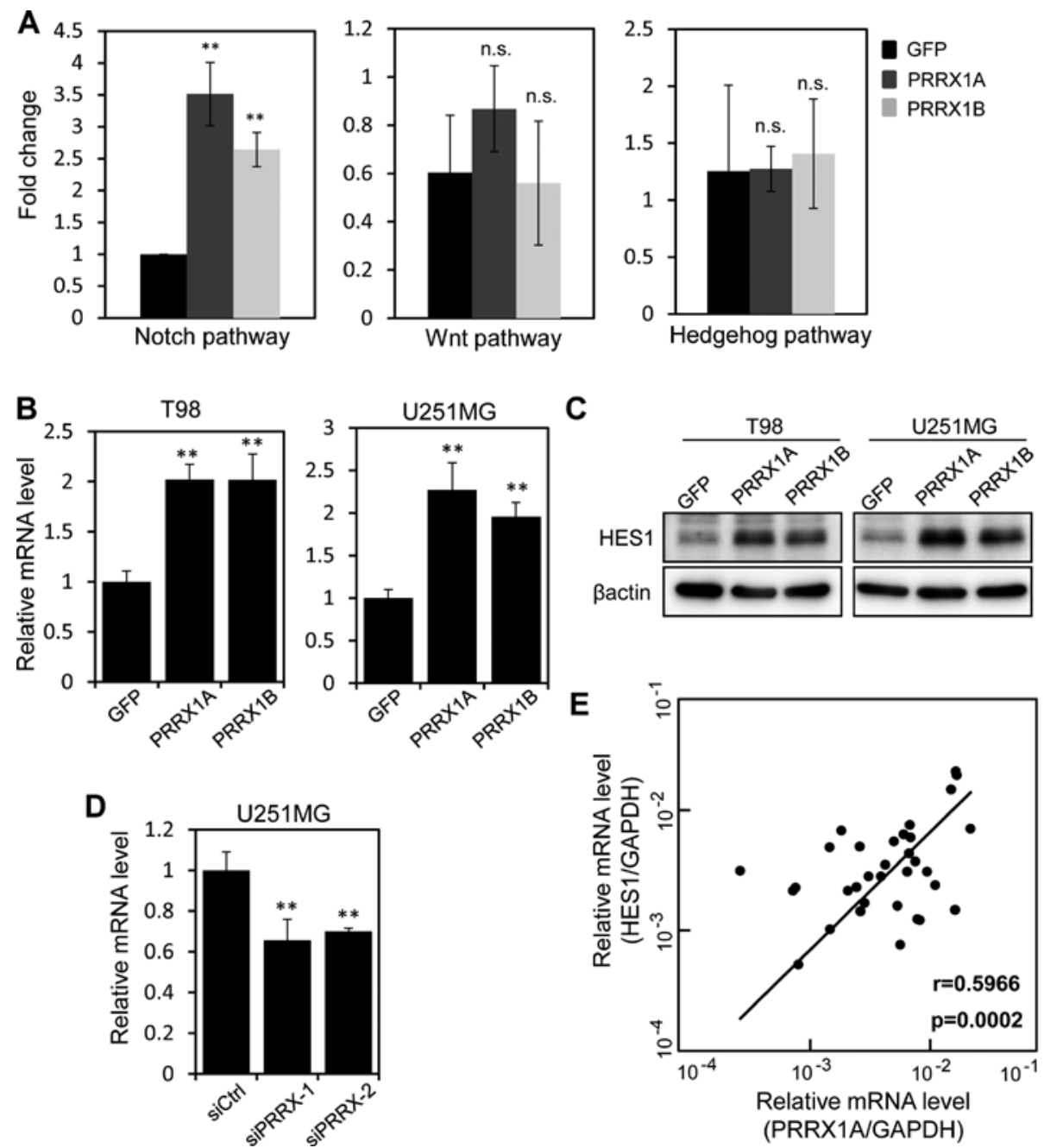

Figure 4. PRRX1 activates Notch signaling. (A) GFP- or GFP-PRRX1-expressing T98 cells were transfected with a reporter plasmid for each signaling pathway together with pRK-Luc to normalize for transfection efficiency. Twenty-four hours later, the cells were lysed, and luciferase activities were measured. The graphs indicate the relative activities of luciferase. Three independent experiments were performed, and the data are shown as the means $\pm \mathrm{SD}\left({ }^{* *} \mathrm{P}<0.01\right.$, n.s., not significant). (B) Level of HES1 mRNA in each cell line was determined by quantitative RT-PCR and normalized to GAPDH. The graphs show the relative levels of the HES1 mRNA. Three independent experiments were performed, and the data are shown as the means \pm SD $\left({ }^{* *} \mathrm{P}<0.01\right)$. (C) GFP- or GFPPRRX1-expressing cells were lysed, and expression of HES1 was examined by immunoblotting. (D) U251MG cells were transfected with two different PRRX1 siRNAs and 3 days later, the level of HES1 mRNA in each cell line was determined by quantitative RT-PCR. Three independent experiments were performed, and the data are shown as the means $\pm \mathrm{SD}\left({ }^{* *} \mathrm{P}<0.01\right)$. (E) Relative expression of the PRRX1A and HES1 mRNAs normalized to GAPDH mRNA in glioma tissues was determined by quantitative RT-PCR. The Pearson's correlation coefficients (r) are shown. PRRX1, paired related homeobox 1.
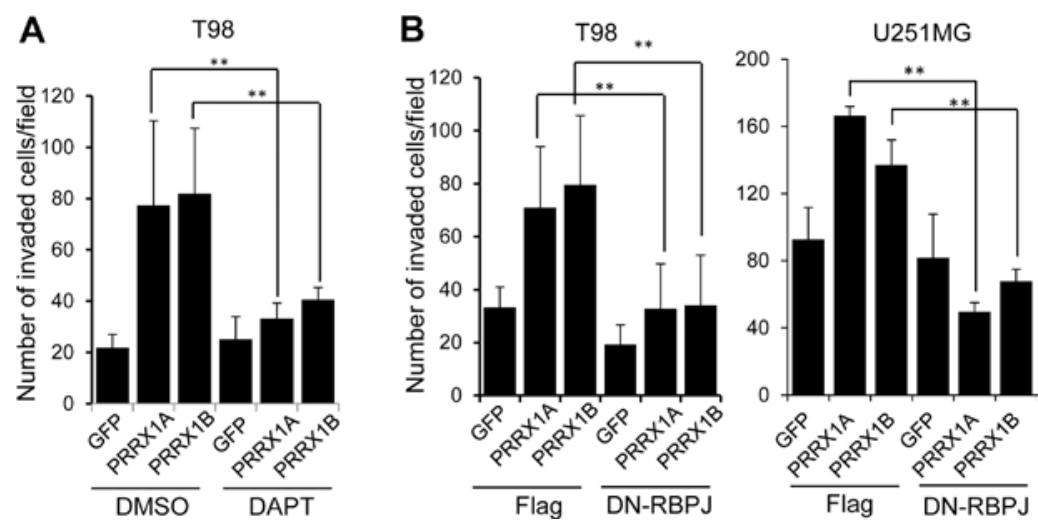

Figure 5. Inhibition of Notch signaling suppresses PRRX1-mediated cell invasion. (A) GFP- or GFP-PRRX1-expressing T98 cells were treated with DMSO or DAPT $(10 \mu \mathrm{M})$ for $12 \mathrm{~h}$ and then subjected to an invasion assay in the presence of DMSO or DAPT. The graph indicates the average number of invaded cells/field. Three independent experiments were performed, and the data are shown as the means $\pm \mathrm{SD}\left({ }^{* * *} \mathrm{P}<0.01\right)$. (B) GFP- or GFP-PRRX1-expressing T98 and U251MG cells were infected with a retrovirus encoding Flag or Flag-DN-RBPJ, and infected cells were selected using neomycin for 5 days. Cells were subjected to an invasion assay, and the graphs indicate the average number of invaded cells/field. Three independent experiments were performed, and the data are shown as the means $\pm \mathrm{SD}\left({ }^{* *} \mathrm{P}<0.01\right)$. PRRX1, paired related homeobox 1 . 
invasion was associated with EMT, we did not observe clear changes in cellular morphology or in the expression of marker proteins, such as vimentin and E-cadherin. These results indicate that the induction of EMT by PRRX1 is dependent on the cell type.

PRRX1 has two isoforms. PRRX1B has an OAR domain at its C-terminal end, while PRRX1A lacks this domain. The exact role of the OAR domain is not yet clear, yet some studies have reported that it is associated with its transcriptional activities (27). PRRX1A deleted of the OAR domain was capable of promoting transcriptional activity, which indicates that the OAR domain has repressive function (28). The OAR domain was also found to attenuate the activity of other transcription factors (29). A previous study showed that PRRX1A and PRRX1B induced different sets of genes, and the expression of PRRX1B is more effective in promoting sphere formation and invasion of pancreatic cells (30). We expressed PRRX1A or PRRX1B in the T98 and U251 cells and examined cell invasion; however, we did not observe any clear differences between them. It has been suggested that the difference in activity of PRRX1A and PRRX1B is mediated by undetermined co-factors; thus, the function of the OAR may differ depending on the cell context.

The Notch signaling pathway is an evolutionarily conserved pathway that regulates differentiation, proliferation and survival. Recent studies have revealed that the Notch signaling pathway is activated in numerous types of cancer. For example, in ovarian cancers, the expression of Notch3 is increased, and inhibition of the Notch pathway inhibits cancer progression (31). In glioblastoma cells, the Notch pathway is important for proliferation, stem cell maintenance and tumorigenesis (32). These studies have clearly shown the important functions of Notch activation for the progression of numerous cancers, including glioblastoma. We showed that PRRX1 expression promoted the activation of Notch signaling, and inhibition of the Notch pathway abolished PRRX1-mediated tumor invasion. In addition, expression levels of PRRX1 and HES1, which is a target gene of the Notch pathway, were correlated in numerous glioma samples. These results suggest a potential role of PRRX1 in the activation of Notch signaling in glioblastoma. PRRX1 is widely expressed, and the Notch pathway is activated in numerous tumors; thus, PRRX1 may regulate Notch activation in various types of tumors.

In summary, we demonstrated that PRRX1 is expressed in glioma and that its inhibition suppresses tumor invasion. We also showed that PRRX1-mediated tumor invasion was dependent on activation of the Notch pathway. Accumulating evidence has clearly shown that PRRX1 is associated with the progression of several types of cancer. Detailed analyses of the functions of PRRX1 may provide novel molecular mechanisms for tumor progression and therapeutic strategies.

\section{Acknowledgements}

We would like to thank Dr Honjo, Dr Sasaki and Dr Moon for the Notch, Hedgehog and Wnt signaling reporter constructs. This study was funded by a grant from the Ministry of Education, Culture, Sports, Science and Technology of Japan (Nanomedicine Molecular Science, 23107010), and from the Takeda Science Foundation.

\section{References}

1. Wen PY and Kesari S: Malignant gliomas in adults. N Engl J Med 359: 492-507, 2008.

2. Bello L, Giussani C, Carrabba G, Pluderi M, Costa F and Bikfalvi A: Angiogenesis and invasion in gliomas. Cancer Treat Res 117: 263-284, 2004.

3. Shah N and Sukumar S: The Hox genes and their roles in oncogenesis. Nat Rev Cancer 10: 361-371, 2010.

4. Coletta RD, Christensen KL, Micalizzi DS, Jedlicka P, VarellaGarcia M and Ford HL: Six 1 overexpression in mammary cells induces genomic instability and is sufficient for malignant transformation. Cancer Res 68: 2204-2213, 2008.

5. McCoy EL, Iwanaga R, Jedlicka P, et al: Six1 expands the mouse mammary epithelial stem/progenitor cell pool and induces mammary tumors that undergo epithelial-mesenchymal transition. J Clin Invest 119: 2663-2677, 2009.

6. Yuan $\mathrm{H}$, Kajiyama $\mathrm{H}$, Ito $\mathrm{S}$, et al: ALX1 induces snail expression to promote epithelial-to-mesenchymal transition and invasion of ovarian cancer cells. Cancer Res 73: 1581-1590, 2013.

7. Costa BM, Smith JS, Chen Y, et al: Reversing HOXA9 oncogene activation by PI3K inhibition: epigenetic mechanism and prognostic significance in human glioblastoma. Cancer Res 70: 453-462, 2010

8. Kern MJ, Argao EA, Birkenmeier EH, Rowe LB and Potter SS: Genomic organization and chromosome localization of the murine homeobox gene Pmx. Genomics 19: 334-340, 1994.

9. Norris RA, Scott KK, Moore CS, et al: Human PRRX1 and PRRX2 genes: cloning, expression, genomic localization, and exclusion as disease genes for Nager syndrome. Mamm Genome 11: 1000-1005, 2000.

10. Martin JF, Bradley A and Olson EN: The paired-like homeo box gene MHox is required for early events of skeletogenesis in multiple lineages. Genes Dev 9: 1237-1249, 1995.

11. Takahashi Y, Sawada G, Kurashige J, et al: Paired related homoeobox 1, a new EMT inducer, is involved in metastasis and poor prognosis in colorectal cancer. Br J Cancer 109: 307-311, 2013.

12. Ocaña $\mathrm{OH}$, Córcoles R, Fabra A, et al: Metastatic colonization requires the repression of the epithelial-mesenchymal transition inducer Prrx1. Cancer Cell 22: 709-724, 2012.

13. Shimozaki K, Clemenson GD Jr and Gage FH: Paired related homeobox protein 1 is a regulator of stemness in adult neural stem/progenitor cells. J Neurosci 33: 4066-4075, 2013.

14. Bass AJ, Watanabe $\mathrm{H}$, Mermel CH, et al: SOX2 is an amplified lineage-survival oncogene in lung and esophageal squamous cell carcinomas. Nat Genet 41: 1238-1242, 2009.

15. Gangemi RM, Griffero F, Marubbi D, et al: $S O X 2$ silencing in glioblastoma tumor-initiating cells causes stop of proliferation and loss of tumorigenicity. Stem Cells 27: 40-48, 2009.

16. Fang X, Yu W, Li L, et al: ChIP-seq and functional analysis of the SOX2 gene in colorectal cancers. OMICS 14: 369-384, 2010.

17. Alonso MM, Diez-Valle R, Manterola L, et al: Genetic and epigenetic modifications of Sox 2 contribute to the invasive phenotype of malignant gliomas. PLoS One 6: e26740, 2011.

18. Ohta S, Mizuno M. Takaoka T and Yoshida J: Augmentation of anti-Fas antibody-mediated apoptosis on human glioma cells by liposomes associated with the antibody. J Neurooncol 35: 7-11, 1997.

19. Kurooka H, Kuroda K and Honjo T: Roles of the ankyrin repeats and C-terminal region of the mouse Notch1 intracellular region. Nucleic Acids Res 26: 5448-5455, 1998.

20. Sasaki H, Hui C, Nakafuku M and Kondoh H: A binding site for Gli proteins is essential for $H N F-3 \beta$ floor plate enhancer activity in transgenics and can respond to Shh in vitro. Development 124: 1313-1322, 1997.

21. Kaykas A, Yang-Snyder J, Héroux M, Shah KV, Bouvier M and Moon RT: Mutant Frizzled 4 associated with vitreoretinopathy traps wild-type Frizzled in the endoplasmic reticulum by oligomerization. Nat Cell Biol 6: 52-58, 2004.

22. Lino MM, Merlo A and Boulay JL: Notch signaling in glioblastoma: a developmental drug target? BMC Med 8: 72, 2010.

23. Mimeault $M$ and Batra SK: Complex oncogenic signaling networks regulate brain tumor-initiating cells and their progenies: pivotal roles of wild-type EGFR, EGFRvIII mutant and hedgehog cascades and novel multitargeted therapies. Brain Pathol 21: 479-500, 2011.

24. Paul I, Bhattacharya S, Chatterjee A and Ghosh MK: Current understanding on EGFR and Wnt/ $\beta$-catenin signaling in glioma and their possible crosstalk. Genes Cancer 4: 427-446, 2013. 
25. Tanigaki $\mathrm{K}$ and Honjo T: Two opposing roles of RBP-J in Notch signaling. Curr Top Dev Biol 92: 231-252, 2010.

26. Thiery JP, Acloque H, Huang RY and Nieto MA: Epithelialmesenchymal transitions in development and disease. Cell 139: 871-890, 2009 .

27. Leussink B, Brouwer A, el Khattabi M, Poelmann RE, Gittenberger-de Groot AC and Meijlink F: Expression patterns of the paired-related homeobox genes MHox/Prx1 and S8/Prx2 suggest roles in development of the heart and the forebrain. Mech Dev 52: 51-64, 1995.

28. Norris RA and Kern MJ: The identification of Prx1 transcription regulatory domains provides a mechanism for unequal compensation by the Prxl and Prx2 loci. J Biol Chem 276: 26829-26837, 2001.
29. Brouwer A, ten Berge D, Wiegerinck R and Meijlink F: The OAR/aristaless domain of the homeodomain protein Cart1 has an attenuating role in vivo. Mech Dev 120: 241-252, 2003.

30. Reichert M, Takano S, von Burstin J, et al: The Prrx1 homeodomain transcription factor plays a central role in pancreatic regeneration and carcinogenesis. Genes Dev 27: 288-300, 2013.

31. Chen X, Thiaville MM, Chen L, et al: Defining NOTCH3 target genes in ovarian cancer. Cancer Res 72: 2294-2303, 2012.

32. Saito N, Fu J, Zheng S, et al: A high Notch pathway activation predicts response to $\gamma$ secretase inhibitors in proneural subtype of glioma tumor-initiating cells. Stem Cells 32: 301-312, 2014 